\title{
DESARROLLO INICIAL EN PLANTACIONES DE Ziziphus thyrsiflora Benth, Geoffroea spinosa Jacq Y Handroanthus chrysanthus Jacq. EN JIPIJAPA, MANABÍ, ECUADOR
}

\author{
AUTORES: Jesús de los Santos Pinargote $\mathrm{Choez}^{1}$ \\ Kiara Scarlett Quiñonez Hurtado ${ }^{2}$ \\ Otto Francisco Mero Jalca ${ }^{3}$ \\ Marcos Pedro Ramos Rodríguez ${ }^{4}$ \\ César Alberto Cabrera Verdesoto ${ }^{5}$
}

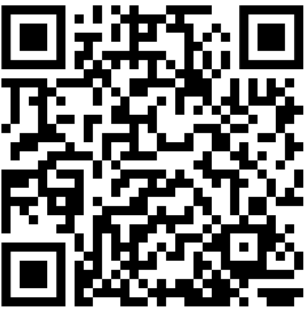

DIRECCIÓN PARA CORRESPONDENCIA: (,jesus.pinargote@ unesum.edu.ec)

Fecha de recepción: 10/01/2019

Fecha de aceptación: 19/04/2019

\section{RESUMEN}

Las especies nativas, en su mayoría, tienen bajas tasas de crecimiento, presentan desarrollo heterogéneo, son sensibles a condiciones de estrés en el establecimiento y son de rendimiento tardío. El objetivo de este trabajo fue evaluar el desarrollo inicial en plantaciones de Ziziphus thyrsiflora Benth, Geoffroea spinosa Jacq y Handroanthus chrysanthus Jacq. en Jipijapa, Manabí, Ecuador. Para el ensayo se utilizó un diseño experimental completamente al azar con arreglo factorial 3 × 2 correspondiendo a especies y a fertilización, con 6 tratamientos y 3 repeticiones logrando un total de 18 unidades experimentales. Como resultados se obtuvo que Ziziphus thyrsiflora mostró el mayor incremento mensual en altura, diámetro y numero de ramas tanto con fertilizante como sin fertilizante. Los valores medios obtenidos con la aplicación de fertilizantes fueron superiores en todos los indicadores lo cual indica la respuesta favorable de las tres especies al fertilizante utilizado, independientemente de que no siempre esta diferencia fue estadísticamente significativa.

PALABRAS CLAVE: Altura, diámetro, fertilización.

\section{INITIAL DEVELOPMENT IN PLANTATIONS OF Ziziphus thyrsiflora Benth, Geoffroea spinosa Jacq and Handroanthus chrysanthus Jacq. IN JIPIJAPA, MANABÍ, ECUADOR}

\section{ABSTRACT}

The native species, in their majority, have low rates of growth, present heterogeneous development, are sensitive to conditions of stress in the establishment and are of late performance. The objective of this work was to evaluate the

\footnotetext{
${ }^{1}$ Ing. Forestal, MSc., Docente Titular Principal 2, Universidad Estatal del Sur de Manabí, Ecuador,

${ }^{2}$ Ing. Forestal, Universidad Estatal del Sur de Manabí, Ecuador,

${ }^{3}$ Ing. Forestal, MSc., Docente Titular Principal 2, Universidad Estatal del Sur de Manabí, Ecuador,

${ }^{4}$ Ing. Forestal, Dr.C., Docente Titular Principal 1, Universidad Estatal del Sur de Manabí, Ecuador,

${ }^{5}$ Ing. Forestal, MSc., Docente Auxiliar Principal 1, Universidad Estatal del Sur de Manabí, Ecuador,
} 
Pinargote, Ch. Jesus, de los Snatos.

initial development in plantations of Ziziphus thyrsiflora Benth, Geoffroea spinosa Jacq and Handroanthus chrysanthus Jacq. in Jipijapa, Manabí, Ecuador. For the trial, a completely randomized experimental design with $3 \mathrm{x}$ 2 factorial arrangement corresponding to species and to fertilization was used, with 6 treatments and 3 repetitions achieving a total of 18 experimental units. As a result, it was obtained that Ziziphus thyrsiflora showed the greatest monthly increase in height, diameter and number of branches, both with fertilizer and without fertilizer. The average values obtained with the application of fertilizers were higher in all the indicators, which indicates the favorable response of the three species to the fertilizer used, independently of the fact that this difference was not always statistically significant.

\section{INTRODUCCIÓN}

La importancia de los bosques en el contexto del desarrollo sostenible es bien reconocida. De acuerdo con la FAO (2018), los bosques y los árboles respaldan la agricultura sostenible debido a que, entre otras cosas, estabilizan los suelos y el clima, regulan los flujos de agua y proporcionan sombra, refugio y un hábitat a los polinizadores y los depredadores naturales de plagas agrícolas. Cuando se integran en los territorios agrícolas, los bosques y los árboles permiten aumentar la productividad de la agricultura. También ayudan a proporcionar seguridad alimentaria a cientos de millones de personas, para quienes constituyen importantes fuentes de alimentos, energía e ingresos durante épocas difíciles.

Independientemente de lo anterior (FAO, 2018) entre 1990 y 2015, la superficie forestal del planeta disminuyó del $31,6 \%$ de la superficie terrestre mundial al 30,6\%, pero el ritmo de pérdida se ha ralentizado en los últimos años. Esta pérdida tiene lugar principalmente en países en desarrollo, en particular en el África subsahariana, América Latina y Asia sudoriental. En el caso de Ecuador, entre los años 1990 y 2015 (FAO, 2015) la deforestación alcanzó la cifra de 2083 000,00 ha de bosques.

En el contexto descrito anteriormente, es importante el establecimiento de nuevas plantaciones forestales. Según García, Sotomayor, Silva, y Valdebenito (2000), la creación de nuevos recursos forestales, a través de plantaciones establecidas por el hombre, puede contribuir positivamente a recuperar terrenos que, por condiciones socioeconómicas, tecnológicas o inadecuada utilización, se encuentran abandonados, sin el menor uso productivo o en proceso de desertificación; y, a la generación de riqueza para beneficio de sus propietarios. La incorporación de especies forestales madereras permitiría aumentar las posibilidades de trabajo de los propietarios, incrementar la productividad del suelo, mejorar en alguna medida la situación económica con la venta de productos que puede obtener de estas plantaciones, y por supuesto, la protección y conservación de los recursos edáficos, hídricos, de vegetación y fauna presentes en su propiedad.

Una de las labores culturales que garantiza el éxito de las plantaciones es la fertilización. Esta actividad, según Albaugh, Rubilar, Alvarez y Allen (2004) y Corporación Nacional Forestal (2013); citados por León-Sánchez, 
Reyes-Pozo, Herrero-Echevarría y Pérez-León (2016), busca mejorar la supervivencia y progreso apropiado de la planta, debido a que estimula el desarrollo de sus raíces, optimiza el uso eficiente del agua con la captación de nutrientes de manera eficaz y suficiente para asegurar su supervivencia y crecimiento inicial, lo que garantiza una ocupación óptima del suelo.

En correspondencia con la problemática tratada anteriormente, esta investigación se desarrolló con el objetivo de evaluar el desarrollo inicial en plantaciones de Ziziphus thyrsiflora Benth, Geoffroea spinosa Jacq y Handroanthus chrysanthus Jacq. en Jipijapa, Manabí, Ecuador.

\section{MATERIALES Y MÉTODOS}

\section{Caracterización del área experimental}

El trabajo se realizó en el jardín forestal de la Universidad Estatal del Sur de Manabí, campus "Los Ángeles", km 1 $1 / 2$ de la vía Jipijapa - Noboa; entre las coordenadas: UTM 1²0'58" latitud Sur y 80³3'51" longitud Oeste (Figura 1). En el área donde se realizó la plantación el suelo fue previamente removido con maquinarias pesadas para establecer un sistema de terrazas.

El clima predominante de Jipijapa es cálido seco en la zona oeste y cálido húmedo con temporadas secas en la zona este, con una temperatura media de $25^{\circ} \mathrm{C}$, afectada por la presencia de dos estaciones: invierno (mayo a octubre) y verano (noviembre a abril). Los valores más altos de humedad y temperatura se registran en el mes de marzo, donde se alcanza una temperatura media del aire de $28{ }^{\circ} \mathrm{C}$. La precipitación promedio anual es de $670 \mathrm{~mm}$, concentrándose la mayor cantidad entre los meses de febrero y marzo. La altitud del sitio es de $320 \mathrm{~m}$. snm.

\section{Especies forestales objeto de estudio}

Handroanthus chrysanthus (Jacq.) S.O. Grose (Guayacán) es un árbol nativo de la Costa, Andes y Amazonía. Crece en las provincias Bolívar, Chimborazo, El Oro, Esmeraldas, Guayas, Loja, Los Ríos, Manabí, Morona Santiago, Napo, Pastaza, Sucumbíos y Pichincha, entre 0 y $2000 \mathrm{msnm}$. La densidad de la madera es de $0,87 \mathrm{gr} / \mathrm{cm}^{3}$ y su color es pardo amarillento claro. Árbol caducifolio que alcanza entre 12 y $20 \mathrm{~m}$ de altura y de 20 a $40 \mathrm{~cm}$ de diámetro. Fuste recto, escasamente ramificado, copa amplia, extendida e irregular. Corteza fisurada pardo-oscuro. Fuste cilíndrico, copa amplia extendida e irregular. Hojas palmadas compuestas, opuestas, ápice agudo y bordes aserrados, de 5 foliolos, de 6 a $12 \mathrm{~cm}$ de longitud, envés áspero y ligeramente pubescente por el envés. Flor tubular, $5 \mathrm{~cm}$ de longitud, con pedúnculo, cáliz de cinco sépalos cafés; corola de cinco pétalos amarillos, en inflorescencia racimosa. El fruto es una cápsula cilíndrica pubescente (parecida a una vaina) de 15 a $30 \mathrm{~cm}$ de longitud, verde (tierna) y café (madura), contiene abundantes semillas aladas. Florece dos veces en el año en junio-julio y noviembre-diciembre. Se propaga por semilla y es de lento crecimiento (Aguirre, 2012). 
Pinargote, Ch. Jesus, de los Snatos.

Ziziphus thyrsiflora Benth (Ébano) se distribuye en la región tumbesina desde la costa del Ecuador hasta el noreste de Perú. Crece en bosques secos y muy secos del litoral ecuatoriano, entre 0 y $500 \mathrm{msnm}$, en las provincias de Loja, El Oro, Guayas y Manabí. Árbol perennifolio de 10 a $18 \mathrm{~m}$ de alto. Fuste muy irregular y variable. Corteza arrugada, color marrón claro a oscuro. En edad adulto presenta nudos en el tallo. Copa redondeada muy densa. Ramas dispuestas en zigzag, con espinas opuestas (pareadas) en las ramitas terminales. Hojas simples alternas, dísticas, glabras, coriáceas de forma ovada-redondeadas con tres nervios principales (trinervadas) bien diferenciados que van desde la base hacia el ápice, borde con dientes ondulados (crenado), pecíolo delgado, haz verde amarillento lustroso y envés verde claro. Flores en inflorescencia cimosa de 1,5 a $4 \mathrm{~cm}$ de longitud, nacen en la base de la hoja, compuesta por pocas flores, fragante, amarillo verdosas. El fruto es una drupa redonda de 1 a $2 \mathrm{~cm}$ de diámetro, caféverdosa (amarillenta) con pedúnculos cortos. Se propaga por semilla y es de crecimiento lento (Aguirre, 2012).

Geoffroea spinosa Jacq. (Seca) habita en laderas, planicies, hondonadas del bosque seco. Crece entre 0 y 2000 msnm, en las provincias de Bolívar, Chimborazo, El Oro, Esmeraldas, Guayas, Loja, Los Ríos, Manabí, Morona Santiago, Napo, Pastaza, Pichincha y Sucumbíos. Árbol caducifolio, frondoso, de $20 \mathrm{~m}$ de altura y $40 \mathrm{~cm}$ de diámetro. Fuste cilíndrico, generalmente recto. Corteza negruzca, muy fisurada en forma de tabiques, presencia de espinas en las ramas. Hojas compuestas paripinnadas, foliolos elípticos grandes de 3,5 de largo y $2 \mathrm{~cm}$ de ancho. Flores pequeñas vistosas de color anaranjado en inflorescencias pequeñas en racimo. Fruto una drupa elipsoidal, carnosa, velluda, de 3-4 cm de largo por 2-2,5 cm de diámetro, de color verde-grisáceo (tierno) y amarillenta (maduro); posee una sola semilla (Aguirre, 2012).

Las especies forestales Ziziphus thyrsiflora Benth, Geoffroea spinosa Jacq y Handroanthus chrysanthus Jacq pueden encontrarse en los bosques secos pluvioestacional en las provincias del Oro, Loja, Esmeraldas, Guayas y Manabí (Aguirre, 2012).

\section{Diseño experimental y toma de datos}

Se utilizó un diseño experimental completamente al azar utilizándose la técnica del experimento bifactorial 3 x 2 (especies y fertilización) para un total de 6 tratamientos, estableciéndose 3 repeticiones para un total de 18 unidades experimentales. Se seleccionaron tres parcelas por cada especie evaluada, escogiéndose 16 plantas en cada parcela, eligiendo ocho plantas al azar aplicándose fertilizantes a cuatro de ellas. La variable dependiente fue el desarrollo inicial de las especies Ziziphus thyrsiflora Benth, Geoffroea spinosa Jacq y Handroanthus chrysanthus Jacq, operacionalizada a través de los indicadores altura, diámetro y número de ramas, mientras que la variable independiente o factor utilizado fueron las condiciones con y sin fertilizantes (F1 con fertilizante $-72 \mathrm{mg}$, F2 sin fertilizante $-0 \mathrm{mg}$ ).

La altura se determinó utilizando un flexómetro, midiendo el crecimiento vertical desde el cuello de la raíz hasta el ápice de las plantas. Para determinar el diámetro se utilizó un calibrador vernier. La medida del crecimiento en diámetro fue tomada a $10 \mathrm{~cm}$ de altura a partir del cuello de la raíz de la planta. El número de ramas se obtuvo a

76 UNESUM-Ciencias. Publicación cuatrimestral. Vol. 3, Año 2019, No. 1 (abril) 
DESARROLLO INICIAL EN PLANTACIONES DE Ziziphus thyrsiflora Benth

través del conteo de las mismas en cada planta en forma ascendente.

Durante el experimento se aplicó riego empleando regaderas manuales. Se aplicaron dos riegos semanales aplicándose dos litros de agua a cada planta durante las mañanas.

\section{Propiedades químicas del suelo}

El análisis químico del suelo se realizó en el Laboratorio de Suelos, Tejidos Vegetales y Aguas de la Estación Experimental "Pichilingue" de la ciudad de Quevedo, por medio de una muestra compuesta de tres submuestras tomadas en el área de estudio que incluyeron: $\mathrm{pH}$ en agua, fosforo disponible en extracto Olsen; potasio, calcio y magnesio, extraíbles en acetato de amonio a pH 4,2; hierro, manganeso, cobre y zinc, extraíbles en acetato de amonio- APTA y boro, en extracto $\mathrm{CaCl}_{2}$ a $0,15 \%$ en ebullición por cinco minutos. También se obtuvieron los valores de materia orgánica.

\section{Incremento medio mensual}

Para determinar el incremento medio mensual del desarrollo inicial de las plantas de las especies objeto de estudio, se utilizaron los valores medios de cada indicador al inicio del experimento y siete meses después, es decir, al finalizar el mismo. El valor obtenido de la resta de este último valor menos el primero, fue dividido por siete, número de meses durante los cuales se desarrolló el experimento.

\section{Efectos de la fertilización}

Para analizar el efecto de la fertilización en el desarrollo inicial de las especies objeto de estudio durante un periodo de siete meses, se utilizaron los valores medios de la última medición realizada en plantas con y sin fertilización, aplicándose a los mismos la prueba t de Student para comparación de medias independientes. Para comparar las varianzas se utilizó la prueba de Levene ya que la misma no asume normalidad. La base de datos se creó con el Microsoft Excel. Los análisis estadísticos se realizaron con el SPSS v.22 (Statistical Package for the Social Sciences), trabajándose con un nivel de significación del 0,05.

\section{RESULTADOS Y DISCUSIÓN}

\section{Propiedades químicas del suelo}

En la Tabla 1 se presentan los resultados del análisis químico del suelo, según lo cual el mismo es alcalino siendo bajos los valores obtenidos para la materia orgánica, el nitrógeno y el potasio mientras que para el fosforo se obtuvo un valor medio. Estos resultados indican la presencia de un suelo de poca fertilidad. 
Tabla 1. Resultados del análisis químico del suelo

\begin{tabular}{lll}
\hline Parámetros & Valores & Unidades \\
\hline $\mathrm{pH}$ & 7,4 & \\
M.O. & 4,9 & $\%$ \\
N. Total & 0,15 & $\%$ \\
$\mathrm{P}$ & 27 & $\mathrm{Ppm}$ \\
$\mathrm{K}$ & 1,29 & $\mathrm{meq} / 100 \mathrm{ml}$ \\
$\mathrm{Ca}$ & 24 & $\mathrm{meq} / 100 \mathrm{ml}$ \\
$\mathrm{Mg}$ & 4,2 & $\mathrm{meq} / 100 \mathrm{ml}$ \\
$\mathrm{Fe}$ & 14 & $\mathrm{Ppm}$ \\
$\mathrm{Mn}$ & 9,1 & $\mathrm{Ppm}$ \\
$\mathrm{Cu}$ & 3,2 & $\mathrm{Ppm}$ \\
Zinc & 1,6 & $\mathrm{Ppm}$ \\
Boro & 0,36 & $\mathrm{Ppm}$ \\
\hline
\end{tabular}

Según Montalvo (2013), los indicadores químicos del suelo presentan condiciones que afectan la relación sueloplanta, disponibilidad de agua y nutrientes a la planta, entre otros. Los indicadores que reflejan condiciones de fertilidad son el pH, la materia orgánica, el nitrógeno, el fosforo y el potasio, por lo que resultan importantes en términos de producción de plantas.

De acuerdo con Pérez, Chango, Fuego, Castillo, Igarza, y Véliz (2014), en el trabajo realizado el valor del Calcio y Magnesio corresponden al total de nutrimentos que está disponible en las plantas: el Calcio $(24 \mathrm{meq} / 100 \mathrm{ml}) \mathrm{se}$ encuentra en un nivel superior al intervalo optimo; y el Magnesio $(4,2 \mathrm{meq} / 100 \mathrm{ml})$ se encuentra en un nivel óptimo. El potasio $(1,29 \mathrm{meq} / 100 \mathrm{ml})$ tiene un nivel óptimo en relación con la media.

Pérez, et al. (2014), asegura que el contenido de cationes mayores en el suelo: Calcio, Magnesio y Potasio, si se encuentran en desequilibrio, a pesar de estar en cantidades relativamente altas, ofrecen malas posibilidades de absorción de los mismos. En el análisis realizado, la relación catiónica $\mathrm{Ca} / \mathrm{Mg}$ posee 5,7; valor que es inferior al balance óptimo. La relación $\mathrm{Mg} / \mathrm{K}$ con un valor de 3,26 se encuentra ligeramente balanceada y la relación $\mathrm{Ca}+$ $\mathrm{Mg} / \mathrm{K}$ cuyo valor es 21,86 se encuentra bien balanceada, es decir, que dos relaciones catiónicas se encuentran en balance óptimo. La sumatoria de bases de los meq/100ml de Ca, $\mathrm{Mg}$ y K, es el índice más directo de las potencialidades nutricionales del suelo. En el estudio existe una buena capacidad de nutrientes del suelo.

Las deficiencias de fosforo se asocia con el $\mathrm{pH}$ y con los contenidos de Al, Fe y Ca y propician la formación de fosfatos de calcio (Pérez et al., 2013). Los elementos menores como el Fe (14 ppm) se encuentran en rango bajo, pero en el intervalo optimo y el $\mathrm{Cu}(3,2 \mathrm{ppm})$, de igual manera, se encuentra bajo, pero dentro del intervalo óptimo.

78 UNESUM-Ciencias. Publicación cuatrimestral. Vol. 3, Año 2019, No. 1 (abril) 
DESARROLLO INICIAL EN PLANTACIONES DE Ziziphus thyrsiflora Benth

La materia orgánica (4.9\%) en el suelo arcillosos- limoso implica un requerimiento de un buen suplemento de nutrientes.

\section{Incremento medio mensual}

Los valores del incremento medio mensual de las tres especies objeto de estudio para los indicadores del desarrollo inicial de las mismas se muestra en la Tabla 2. Se observa que en todos los casos, tanto con fertilizante como sin fertilizante, la especie de mayor incremento mensual fue la $Z$. thyrsiflora seguida de la $G$. spinosa y por último la $H$. chrysanthus.

Tabla 2. Valores del incremento medio mensual con y sin fertilización para altura, diámetro y número de ramas

\begin{tabular}{ccccc}
\hline \multirow{2}{*}{ Especies } & Factores & Altura & Diámetro (cm) & $\begin{array}{c}\text { Número de } \\
\text { ramas }\end{array}$ \\
\cline { 3 - 5 } & & $(\mathbf{c m})$ & 0,26 & 2,57 \\
\multirow{2}{*}{ Ziziphus thyrsiflora } & Con fertilizantes & 27,21 & 0,26 & 2,33 \\
& Sin fertilizantes & 25,27 & 0,18 & 1,95 \\
& Con fertilizantes & 16,94 & 0,13 & 1,24 \\
\hline \multirow{2}{*}{ Geoffroea spinosa } & Sin fertilizantes & 12,79 & 0,10 & 1,48 \\
Handroanthus & Con fertilizantes & 1,60 & 0,05 & 1,00 \\
\hline
\end{tabular}

Muñoz, Erazo y Armijos (2014), en su estudio sobre evaluación de una plantación de tres especies tropicales de rápido crecimiento; a los dos años, al aplicar la prueba de Tukey, encontró diferencias significativas entre las especies, por lo que se considera que las condiciones ecológicas del sitio y los requerimientos climáticos deben ser similares a los de su habitad natural para obtener un mejor desarrollo; este ensayo se los realizo en suelos de textura arcillosa y el resultado se lo atribuye a las características fisiológicas y genéticas de las especies del ensayo.

\section{Efectos de la fertilización}

La fertilización temprana en la etapa del establecimiento, es una medida silvicualtural que contribuye al adecuado crecimiento inicial de los árboles, puesto que en suelos degradados por efectos de la erosión, generalmente las limitaciones nutricionales pueden ser causa de insuficiente crecimiento y de predisposición a daños bióticos y abióticos para las plantas.

La Tabla 3 muestran los valores medios obtenidos con y sin fertilización para altura, diámetro y número de ramas de las especies Z. thyrsiflora, G. spinosa y $H$. chrysanthus. Se observa que los valores medios obtenidos con la aplicación de fertilizantes fueron superiores en todos los indicadores lo cual indica la respuesta favorable de las tres especies al fertilizante utilizado. No obstante, no siempre esta diferencia fue estadísticamente significativa. 
Tabla 3. Valores medios con y sin fertilización para altura, diámetro y número de ramas

\begin{tabular}{ccccc}
\hline \multirow{2}{*}{ Especies } & Factores & \multicolumn{3}{c}{ Indicadores } \\
\cline { 3 - 5 } & & $\begin{array}{c}\text { Altura } \\
(\mathbf{c m})\end{array}$ & Diámetro $(\mathbf{c m})$ & $\begin{array}{c}\text { Número de } \\
\text { ramas }\end{array}$ \\
\hline \multirow{2}{*}{ Ziziphus thyrsiflora } & Con fertilizantes & 202,08 & 3,26 & 38,50 \\
& Sin fertilizantes & 174,33 & 2,53 & 28,25 \\
\hline \multirow{2}{*}{ Geoffroea spinosa } & Con fertilizantes & 184,92 & 2,59 & 22,58 \\
& Sin fertilizantes & 144,42 & 2,13 & 18,25 \\
\hline Handroanthus & Con fertilizantes & 35,67 & 1,33 & 12,92 \\
chrysanthus & Sin fertilizantes & 33,63 & 1,08 & 10,67 \\
\hline
\end{tabular}

León et. al. (2016) señalaron que una buena fertilización debe considerar todos los factores de fertilidad del suelo, puesto que si se fertiliza tan solo considerando el régimen nutritivo se podría encontrar resultados nulos e incluso negativos para el crecimiento de las especies en estudio.

Solla-Guyón et al. (2004) comunican resultados positivos en altura y diámetro ante la fertilización con cenizas de biomasa en plantaciones forestales jóvenes.

Donoso et al. (2007), al evaluar el efecto de la fertilización y las malezas en el desarrollo inicial de una plantación de Nothofagus dombeyi, encontraron que finalizada la primera temporada de crecimiento, la fertilización afectó significativamente el diámetro a la altura del cuello y el factor de productividad, pero no la altura total.

En la Tabla 4 se muestran los resultados de la prueba $t$ para muestras independientes aplicada a los valores obtenidos con y sin fertilización para los indicadores altura, diámetro y número de ramas correspondientes a la variable dependiente desarrollo inicial de las especies Z. thyrsiflora, G. spinosa y H. chrysanthus. En primer lugar, el contraste de Levene $(F)$ indica que las varianzas poblacionales son iguales $(p>0,05)$ en todos los casos. Los niveles críticos bilaterales asociados al estadístico $t$ permiten concluir que las medias de los distintos indicadores con y sin fertilizantes no son iguales ( $\mathrm{p}<0,05$ ), excepto para el caso de la altura en la especie $H$. chrysanthus y del número de ramas.

80 UNESUM-Ciencias. Publicación cuatrimestral. Vol. 3, Año 2019, No. 1 (abril) 
Tabla 4. Resultados de la comparación de medias utilizando la prueba t para muestras independientes

\begin{tabular}{|c|c|c|c|c|c|c|}
\hline \multirow{2}{*}{ Especies } & \multirow{2}{*}{ Indicadores } & \multicolumn{2}{|c|}{$\begin{array}{l}\text { Prueba de Levene de } \\
\text { calidad de varianza }\end{array}$} & \multicolumn{3}{|c|}{ Prueba t para la igualdad de medias } \\
\hline & & $\mathbf{F}$ & Sig. & $\mathbf{t}$ & gl & Sig. (bilateral) \\
\hline \multirow{3}{*}{$\begin{array}{l}\text { Ziziphus } \\
\text { thyrsiflora }\end{array}$} & Altura & 0,196 & 0,662 & 2,181 & 22 & 0,040 \\
\hline & Diámetro & 0,141 & 0,711 & 2,732 & 22 & 0,012 \\
\hline & Número de ramas & 0,070 & 0,794 & 3,130 & 22 & 0,005 \\
\hline \multirow{3}{*}{$\begin{array}{l}\text { Geoffroea } \\
\text { spinosa }\end{array}$} & Altura & 0,065 & 0,801 & 2,455 & 22 & 0,022 \\
\hline & Diámetro & 0,564 & 0,461 & 2,434 & 22 & 0,024 \\
\hline & Número de ramas & 0,041 & 0,841 & 1,114 & 22 & 0,277 \\
\hline \multirow{3}{*}{$\begin{array}{l}\text { Handroanthus } \\
\text { chrysanthus }\end{array}$} & Altura & 0,003 & 0,958 & 0,515 & 22 & 0,611 \\
\hline & Diámetro & 0,900 & 0,353 & 2,595 & 22 & 0,017 \\
\hline & Número de ramas & 0,035 & 0,854 & 1,650 & 22 & 0,113 \\
\hline
\end{tabular}

En un estudio sobre efectos de diferentes prácticas de preparación del terreno y fertilización sobre el crecimiento inicial del Pinus spp en el NE argentino, Fernández, Rodríguez, Lupi, Hernandez y Reis (1999), encontraron que el Pinus taeda implantado sobre camellones manifestó respuestas positivas a la adición de fertilizante NPK.

Balám, et. al. (2015), estudiando la fertilización inicial de plantaciones comerciales de teca en un experimento factorial para evaluar el efecto de N. P. K. sobre el crecimiento inicial, con dosis de testigos medias y altas o bajas, según se encontrara arriba o abajo la dosis; los resultados determinaron que con la dosis alta de nitrógeno, no se observó efecto en el crecimiento por la aplicación de fosforo; contrario a los esperado, el $\mathrm{K}$ influyo negativamente en el crecimiento del área basal. Los resultados sugieren que la aplicación alta de K puede afectar la absorción de otros nutrientes, aunque este no es el caso del presente estudio.

En un estudio sobre la respuesta del pachaco (Schizolobium parahybum Vell. Conc) y la melina (Gmelina arbórea Roxb.) a la aplicación de biocarbón y fertilización en el sur de la amazonia ecuatoriana, Valarezo, et al. (2016), en dos sitios analizados obtuvieron que los efectos de fertilización (N, P, K, Mg, S y Zn más la cal), fueron altamente significativos para las tres variables de las especies arbóreas indicadoras pachaco y melina: altura de planta y diámetro basal y DAP, durante todas las fechas de registro a lo largo de los dos años desde la plantación. En los dos sitios experimentales los valores de altura de planta y de DAP de las dos especies arbóreas indicadoras son notoriamente mayores a aquellas que reportan otros investigadores para éstas en otras partes del mundo.

Vázquez-Cisneros, et al. (2018), en un estudio sobre crecimiento y supervivencia de una plantación encontraron que la aplicación inicial de fertilizantes de liberación lenta 12-25-12, 09-23-14 y 18-06-12 de N-P-K favoreció el crecimiento en altura, diámetro y volumen de los brinzales de Pinus greggii var. greggii en comparación con el 
Pinargote, Ch. Jesus, de los Snatos.

testigo. Los tratamientos de fertilización no influyeron en la supervivencia a un año del establecimiento de la plantación. Con relación a la concentración foliar, solo el fósforo mostró diferencias significativas entre tratamientos. El nitrógeno y fósforo fueron limitantes, pero la aplicación de potasio favoreció el crecimiento aun cuando este elemento se encuentra en el suelo en niveles suficientes.

Hernández, López, y Flores (2018), concluyeron que el nutrimento que más incrementó el diámetro basal fue el fósforo con la dosis alta. El sitio de estudio presenta deficiencia de $\mathrm{P}$, ya que al aumentar su dosis se incrementa el diámetro basal. La aplicación de nitrógeno en la dosis media (4 g árbol-1) genera mayor incremento de altura en los brinzales de Pinus cooperi. El potasio no tiene ningún efecto positivo en las variables evaluadas. Los nutrimentos deficientes son fósforo y nitrógeno. La fertilización no siempre garantiza un mejoramiento de las tasas de crecimiento del árbol; por el contrario, esta práctica puede reducir los rendimientos, cuando se aplica el nutrimento no limitante.

\section{CONCLUSIONES}

La permanencia estudiantil en Educación Superior, debe llegar a ser el sistema de prevención que impulse a las Instituciones de Educación Superior a implementar mecanismos y herramientas útiles, de acuerdo al contexto específico en que se desenvuelven, a acompañar a los estudiantes desde el ingreso hasta el final, con el objetivo de culminar exitosamente el camino emprendido.

Los espacios abiertos al interior de las instituciones, que promueven la discusión y generación de herramientas y recursos, y sobre todo la innovación y adaptación a los nuevos modelos de educación, reducen la deserción estudiantil, siendo esta el resultado de sistemas de permanencia débiles o poco desarrollados al interior de las universidades.

Teniendo en cuenta la variedad y la problemática de los factores involucrados en la permanencia estudiantil, el modelo de la investigación disgregó las dimensiones relacionadas con el fenómeno, para abordarlo desde la visión de los docentes, buscando facilitar el planteamiento de alternativas útiles para la generación de condiciones que garanticen el objetivo final del estudiante.

Los docentes consideran fuera de los factores económicos y laborales, dos situaciones muy relevantes para la permanencia estudiantil: la orientación vocacional, y los imaginarios que traen los estudiantes al ingresar a la Educación Superior.

La labor del docente incide efectivamente en la toma de decisión de un estudiante frente a la posibilidad de desertar. De igual manera las áreas administrativas apoyan la satisfacción de los estudiantes en la institución, ofreciendo valores agregados para permanecer en la misma.

Los docentes manifestaron satisfacción y apoyo académico institucional en cuanto a acompañamiento pedagógico, sin embargo en cuanto a congresos y eventos externos consideran que podría ser mayor el apoyo institucional para mantenerse actualizados y relacionados con el sector productivo.

82 UNESUM-Ciencias. Publicación cuatrimestral. Vol. 3, Año 2019, No. 1 (abril) 
Con el propósito firme de lograr la permanencia estudiantil en las instituciones de Educación Superior, es indispensable disgregar las variables que inciden en el fenómeno, desplegando estrategias para cada una, encontrando resultados pertinentes según la realidad y el contexto institucional. Las variables en mención, están enmarcadas entre las dimensiones retención y persistencia, que sumadas, aportan nociones indispensables para aumentar la permanencia, y disminuir la deserción.

Finalmente, se insiste en la importancia de la creación e implementación, a nivel institucional, de estrategias de evaluación del impacto de los planes en pro de la retención, con el fin de garantizar la efectividad de los procesos y afianzar la estructura interna del proceso generado, para fomentar la permanencia estudiantil.

\section{REFERENCIAS BIBLIOGRÁFICAS}

Aguirre, Z. H. (2012). Especies forestales de los bosques secos del Ecuador. Recuperado de http://dspace.unl.edu.ec/jspui/bitstream/123456789/288/1/ZOFRE\%20AGUIRRE\%20MENDOZA.pdf

Balám, M., Gómez, A., Vargas, J., Aldrete, A., \& Obrador, J. (2015). Fertilización inicial de plantaciones comerciales de teca. Revista Fitotecnia Mexicana, 38(2), 205-212.

Donoso, P. J., Gerding, V., Uteau, D., Soto, D. P., Thiers, O., \& Donoso, C. (2007). Efecto de fertilización y cobertura de malezas en el crecimiento inicial y la mortalidad de una plantación de Nothofagus dombeyi en la Cordillera de Los Andes. Bosque (Valdivia), 28(3), 249-255.

Fernández, R., Rodriguez Aspillaga, F., Lupi, A., Hernández, A., \& Reis, H. (1999). Efectos de diferentes prácticas de preparación del terreno y fertilización sobre el crecimiento inicial del Pinus spp en el NE argentino. Bosque, 20(1), 47-55. Recuperado de https://www.researchgate.net/profile/Roberto_Fernandez12/publication/283781656_Efectos_de_diferentes_ practicas_de_preparacion_del_terreno_y_fertilizacion_sobre_el_crecimiento_inicial_del_Pinus_spp_en_el_ NE_argentino/links/568a7f0908ae1e63f1 fbce24/Efectos-de-diferentes-practicas-de-preparacion-del-terrenoy-fertilizacion-sobre-el-crecimiento-inicial-del-Pinus-spp-en-el-NE-argentino.pdf.

García, E., Sotomayor, A., Silva, S., \& Valdebenito, G. (2000). Establecimiento de plantaciones forestales. Eucalyptus sp. Fondo de Desarrollo e Innovación. Recuperado de http://biblioteca.infor.cl/DataFiles/14148.pdf.

Hernández, R. R., López, M. Á., \& Flores, P. (2018). Crecimiento y estado nutrimental de una plantación de Pinus cooperi Blanco fertilizada con NPK. Revista Mexicana de Ciencias Forestales (9)48. http://dx.doi.org/10.29298/rmcf.v8i48.123.

León-Sánchez, M. A., Reyes-Pozo, J. L., Herrero-Echevarría, G., \& Pérez-León, V. E. (2016). Efecto de la fertilización sobre el crecimiento en diámetro y altura de Pinus caribaea en plantaciones del occidente de Cuba. Madera y bosques, 22(3), 87-101. Recuperado de http://www.scielo.org.mx/scielo.php?script=sci_arttext\&pid=S1405-04712016000300087

Montalvo, C. (2013). Efectos de la contaminación del suelo en la productividad de cinco sectores agrícolas de la parroquia de Tumbaco. Quito: Universidad Central del Ecuador.

Muñoz, J., Erazo, S., \& Armijos, D. (2014). Composición florística y estructura del bosque seco de la quinta experimental "El Chilco" en el suroccidente del Ecuador. Cedamaz, 4(1), 53 - 61.

Organización de las Naciones Unidas para la Alimentación y la Agricultura. (2015). Evaluación de los recursos forestales mundiales 2015 - Compendio de datos. Recuperado de http://www.fao.org/3/a-i4808s.pdf.

Organización de las Naciones Unidas para la Alimentación y la Agricultura. (2018). El estado de los bosques del mundo - Las vías forestales hacia el desarrollo sostenible. Roma. Recuperado de http://www.fao.org/3/I9535ES/i9535es.pdf. 
Pinargote, Ch. Jesus, de los Snatos.

Pérez, N., Chango, R., Fuego, M., Castillo, I., Igarza, U., \& Véliz, J. (2014). Dinámica de crecimiento de plántulasde vitex parviflora juss en sustratos de compost de corteza de las especies Eucalyptus saligna Smith, y Eucalyptus pellita F. Muell en viveros forestales. Revista Cubana de Ciencias Forestales, 2(2), 178-190.

Solla-Guyón, F., Rodríguez, R. y Merino, A. (2004). Respuesta de plantaciones forestales jóvenes sobre suelos ácidos a la fertilización con cenizas de biomasa. Cuadernos de la Sociedad Española de Ciencias Forestales, 20, 227-232.

Valarezo, C., Villamagua, M. A., Mora, R. M., Maza, H., Wilcke, W., \& Nieto, C. (2017). Respuesta del pachaco (Schizolobium parahybum Vell. Conc) y la melina (Gmelina arbórea Roxb.) a la aplicación de biocarbón y fertilización en el sur de la Amazonia Ecuatoriana. Bosques Latitud Cero, 6(1). Recuperado de http://revistas.unl.edu.ec/index.php/bosques/article/viewFile/183/178.

Vázquez-Cisneros, I., Prieto-Ruíz, J. A., López-López, M. A., Wehenkel, C., Domínguez-Calleros, P. A., \& MuñozSáez, F. E. (2018). Growth and survival of a plantation of Pinus greggii Engelm. ex Parl. var. greggii under different fertilization treatments. Revista Chapingo Serie Ciencias Forestales y del Ambiente, 24(2), 251264. doi: 10.5154/r.rchscfa.2017.05.036.

84 UNESUM-Ciencias. Publicación cuatrimestral. Vol. 3, Año 2019, No. 1 (abril) 